
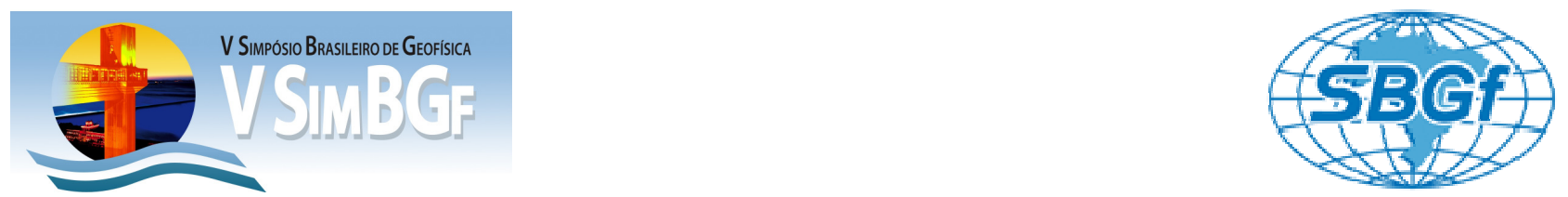

\title{
Utilização de métodos geoelétricos para auxílio ao planejamento de lavra de pedreira de gnaisse
}

Camila Carroci Martins, Vagner Roberto Elis, Universidade de São Paulo

Copyright 2012, SBGf - Sociedade Brasileira de Geofísica

Este texto foi preparado para a apresentação no V Simpósio Brasileiro de Geofísica, Salvador, 27 a 29 de novembro de 2012. Seu conteúdo foi revisado pelo Comitê Técnico do V SimBGf, mas não necessariamente representa a opinião da SBGf ou de seus associados. É proibida a reprodução total ou parcial deste material para propósitos comerciais sem prévia autorização da SBGf.

\section{Abstract}

This work intended to create a geolectrical/geological model in a gneiss mine to assist the project of mine engineering, looking forward to advancing of work front. For that were used electric method, resistivity and induced polarization. We used the vertical electrical sounding (VES) technique to identify thickness of the topsoil, electrical pseudosection (EP) technique to map the lateral contact between differents lithologies, and electromagnetic induction method 34 (EM34), looking for map, with lower resolution, contact between lithologies.

The study area was a mine of gnaisse located near Embu city, São Paulo state, Brazil. This area has resistive rock, gneiss, which comes from granite metamorphism, and a more conductive schist. It is very important to understand the behavior of gnaiss which is exploitated in the mine. Thus thirteen soundings of VES and four profile of EP were acquired. We will acquire two profile of EM34.

So far the VES data that we have processed, allowed to us identify the topsoil thickness and unexpectedly identified, in some area, an anomalous rock. This kind of rock has an intermediate composition and resistivity between gneiss and schist. Until now this rock was named minolitico schist. The EP data mapped contacts between gneiss and schist, favoring the knowledge of behavior rock. Future EM34 acquisition will be made in two profiles that we acquired EP data. If the results between EM34 and EP will be similar, the EM34 may be a primary method of data acquisition because is cheaper and faster.

\section{Introdução}

Neste trabalho a Geofísica se insere no ramo da Geologia de Engenharia, mais especificamente na Geotecnia que segundo Santos (2002) é entendida como o ramo da Engenharia que se ocupa da caracterização e comportamento dos materiais da crosta terrestre para fins de engenharia. Com isso uma operação de engenharia se respalda na concordância entre a solicitação adotada e as características geológicas dos terrenos. Portanto é extremamente importante um diagnóstico prévio o mais perto do exato possível. Neste contexto e aliado ao desenvolvimento sustentável (Mineração e Desenvolvimento Sustentável, 2008) a geofísica se insere como uma ótima técnica de análise a priori ou para continuidade dos trabalhos.

As pedreiras e os depósitos minerais são áreas fontes de diversos materiais agregados como a areia, brita e o cascalho. A lavra é a atividade de extração, exploração e beneficiamento do minério, que transforma a matéria bruta em matéria prima para suprir uma enorme gama de ramos industriais. Estes materiais são usados principalmente na construção civil, sendo o termo "agregados para a construção civil", que consta na legislação mineral para definir essa classe de substâncias minerais (Valverde, 2001). Os materiais agregados têm como principal uso a confecção de concreto, blocos para revestimento de edifícios, proteção de taludes de barragens, pedra britada para os leitos de ferrovias, aeroportos e rodovias, blocos para calçamento de ruas, avenidas, em indústria de cerâmica, de vidro, etc.

A empresa EMBU S/A ENGENHARIA E COMÉRCIO, responsável pela mineração da Pedreira Embu, atua no mercado há 47 anos e trabalha com capital $100 \%$ nacional. A empresa tem três jazidas em São Paulo, Pedreira Embu, Pedreira Itapeti e Pedreira Juruaçu; e uma em Vila Velha no Espírito Santo, Pedreira Rydien Mineração (Embusa, 2012). Este trabalho faz um estudo na Pedreira Embu que se localiza na Estrada Maria José Ferraz Prado, 3100. Bairro Itatuba, Município Embu, São Paulo/SP. O processo de produção aplicado na pedreira pode ser dividido nos seguintes estágios: Decapeamento e remoção de material de transição, Perfuração e desmonte, Carregamento e transporte, Britagem primária, Rebritagem e classificação e Controle de qualidade.

No contexto em que uma pedreira queira avançar sua frente de lavra de uma forma eficaz e sustentável, os métodos geofísicos mais eficientes é o método da eletrorresistividade. Além disso são de rápida aplicação e de baixo custo financeiro quando comparado com sondagens diretas para caracterização da região.

\section{Metodologia/ Problema Investigado}

Neste trabalho foi utilizado o métodos geofísicos da eletrorresistividade e será utilizado o método eletromagnético de baixa frequência 34 . O método da eletrorresistividade busca determinar a resistividade elétrica ou/e polarização induzida do substrato da Terra. Para isso emprega uma corrente artificial injetada no terreno a partir de dois eletrodos denominados eletrodos de corrente A e B. Então, são medidas na superfície as diferenças de potencial, através de dois eletrodos denominados eletrodos de potencial M e N. Embasado nas propriedades elétricas é possível associar os valores da resistividade a diferentes materiais geológicos (Bortoloso, 2011). Este método utilizou as técnicas da sondagem elétrica vertical (SEV), para obter informações de resistividade e profundidade ao longo de um perfil e caminhamento elétrico (CE), para obter a variação lateral das propriedades elétricas a profundidades constantes; 
adquirindo dados de resistividade e polarização induzida, figura 1. A polarização induzida ou Induced Polarization (IP) é um fenômeno elétrico estimulado por corrente. Em alguns materiais geológicos, quando a corrente elétrica para de atravessar o corpo, o campo elétrico produzido pela passagem de corrente não cessa totalmente, mas vai diminuindo lentamente. Este fenômeno é conhecido como polarização induzida ou residual.

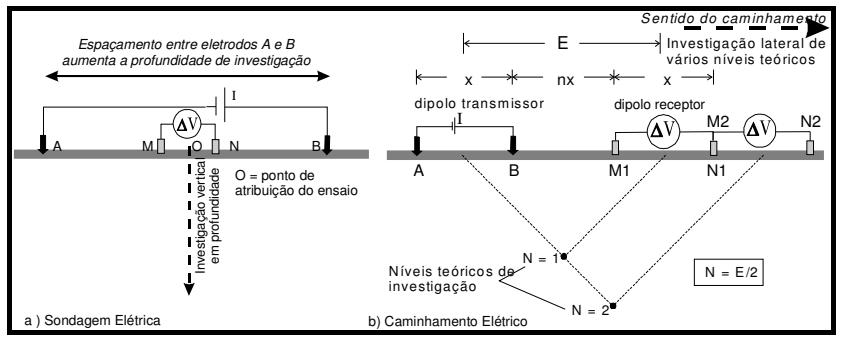

Figura 1 - Configuração de ensaios de campo para as técnicas de Sondagem Elétrica Vertical (item a) e Caminhamento Elétrico (item b), (Retirado de Elis e Zuquette, 2000).

Para os dados de SEV coletados, foi utilizado o eletroresistivimetro Syscal R2 da empresa Iris Instrument, pertencente ao Departamento de Geofísica do IAG/USP. Foram adquiridos 13 dados utilizando a técnica de aquisição Schlumberger, visando identificar a profundidade do capeamento e do topo do gnaisse. Para os dados de CE, foi utilizado o equipamento também o eletroresistivímetro Syscal R2 acoplado com outro aparelho chamado Syscal Pro Ten Channel Resistivity Meter, ambos da Iris Intrument. Foram adquiridos 4 perfis de CE utilizando a técnica dipolo-dipolo para visualizar o contato lateral entre o gnaisse e o xisto. Neste trabalho serão apresentados apenas três dados de SEV e dois dados de CE.

O outro método geofísico utilizado foi o método Eletromagnético Indutivo (EM34) ou método da bobina dupla de fonte móvel, mede a condutividade elétrica em subsuperfície a partir da técnica de baixo número de induções. Este método tem como princípio a passagem de uma corrente elétrica alternada por meio de uma bobina emissora, que resulta em um campo eletromagnético primário $H_{p}$. A propagação deste campo em um meio condutor no subsolo, assumido como meio homogêneo, resulta em correntes secundárias alternadas que produzem um campo eletromagnético secundário $\mathrm{H}_{\mathrm{s}}$, figura 2 (Moreira, 2007).

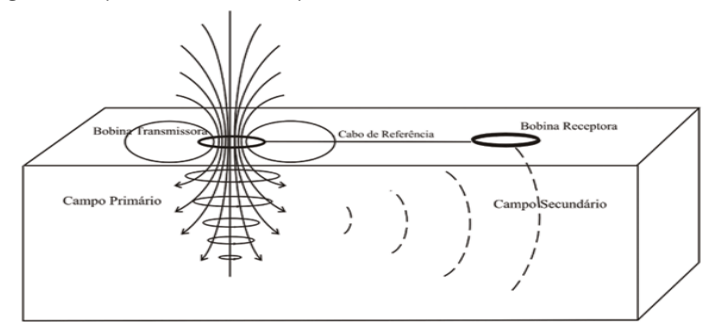

Figura 2: Princípio do Eletromagnético Indutivo 34 (retirado de Moreira, 2007).

\section{Resultados}

Os levantamentos geofísicos de eletrorresistividade foram realizados priorizando bordejar o limite da pedreira, figura $3 \mathrm{em}$ anexo.

Os dados de Sondagem Elétrica Vertical foram processados no programa RESIX-IP. Este é um programa desenvolvido pela Interpex. O RESIX-IP é um programa iterativo de modelagem direta ou inversa para interpretar dados de resistividade e polarização induzida juntos ou separadamente. Após a inversão dos dados, o programa cria um modelo de camadas equivalentes e suaves para os dados de RE e IP, como pode ser visto no processamento dos dados das SEV's 1, 3 e 7, figuras 4 a 6.

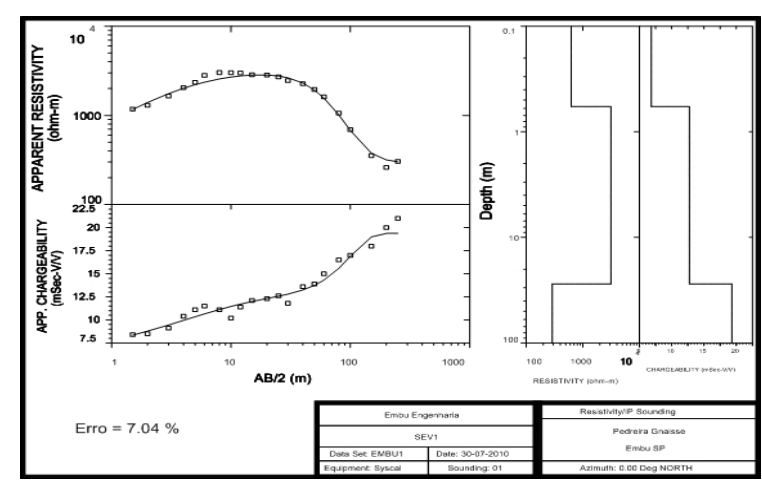

\begin{tabular}{|l|c|c|c|}
\hline & Camada 1 & Camada 2 & Camada 3 \\
\hline Resistividade & 625,68 & 3150,20 & 287,73 \\
\hline Cargabilidade & 6,84 & 12,75 & 19,32 \\
\hline Profundidade & 0,57 & 27,57 & \\
\hline
\end{tabular}

Figura 4: Processamento da SEVI

Esta sondagem esta localizada em uma região com embasamento xistoso, que é uma rocha de resistividade baixa, em torno de 0,1 a 50 Ohm.m (Telford, 1990) e cargabilidade mais alta. Sendo assim, foi possível identificar 3 camadas, a primeira com uma resistividade em torno de 630 Ohm.m que pode ser identificado como um solo de alteração ou um gnaisse alterado. Já a segunda camada apresenta uma resistividade mais alta, o que justificaria associa-la ao gnaisse. Entretanto, a resistividade do gnaisse são costuma ser maior. Assim é razoável associar esta camada com um gnaisse fraturado. Já o embasamento apresenta um resistividade em torno de 300 Ohm.m, o que nos leva a associa-la ao xisto, que portanto começa a uma profundidade de $27,57 \mathrm{~m}$ aproximadamente. É importante comentar que os modelos se ajustaram bem aos dados coletados, principalmente a curva de resistividade. 


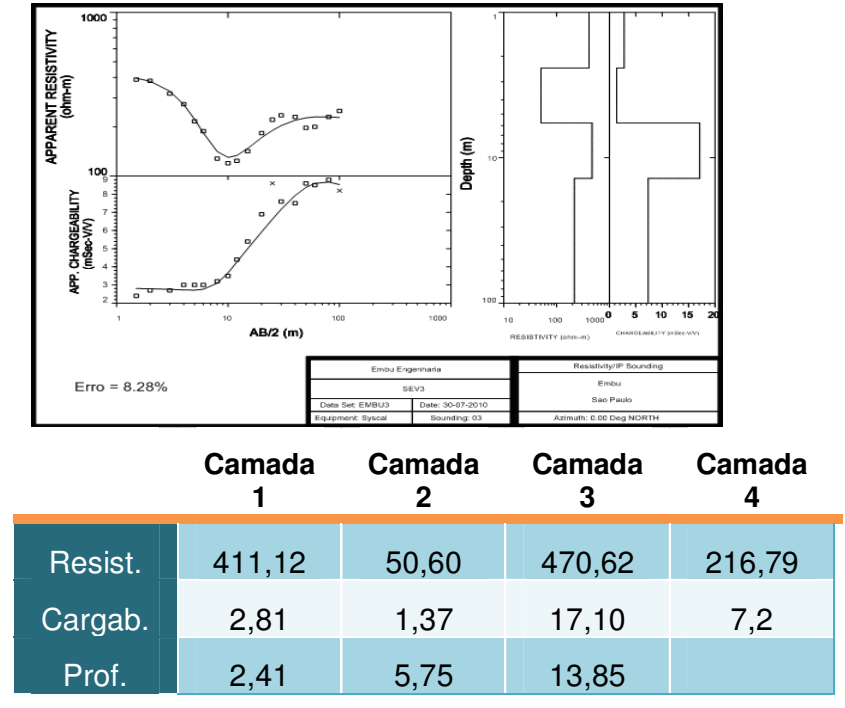

Figura 5: Processamento da SEV 3

Esta SEV esta localizada em uma área crítica, pois seria no suposto contato entre 0 xisto e 0 xisto minolítico. O resultado da SEV apresenta um erro considerado baixo, isto é abaixo de $10 \%$ e comportamento estável. Entretanto foi necessário mascarar dois dados de IP e, com isso, o comportamento da curva teórica ficou melhor. É importante ressaltar que analisando isoladamente a curva de resistividade, seria possível identificar mais que 4 camadas, principalmente em maiores aberturas. Mas como os dados foram processados conjuntamente, considerou-se que a curva de IP apresentar maior legibilidade.

Foi identificado 4 camadas. A primeira com uma resistividade em torno de $400 \Omega$.m e cargabilidade em torno de $3 \mathrm{mSeg} . \mathrm{V} / \mathrm{V}$. Assim seria compatível identifica-la como um solo proveniente do xisto. A segunda apresenta resistividade em torno de $50 \Omega . \mathrm{m}$ e cargabilidade em torno de $1,4 \mathrm{mSeg} . \mathrm{V} / \mathrm{V}$. Com isso, seria plausível identifica-la como um solo mais saturado. A camada 3 apresenta resistividade parecida com a camada 1, mas cargabilidade maior o que nos faz identifica-lo como o xisto minolítico. E a última camada com resistividade e cargabilidade menor que a terceira seria o xisto. Sendo assim o contato estaria a uma profundidade de 13,85 metros.

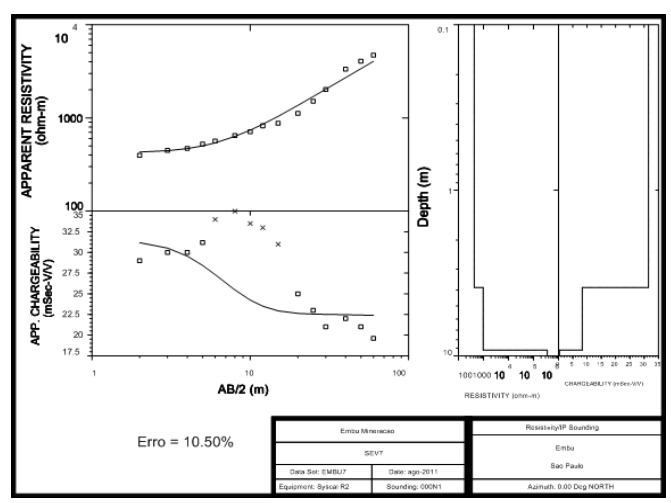

\begin{tabular}{|l|c|c|c|}
\hline & Camada 1 & Camada 2 & Camada 3 \\
\hline Resistividade & 424,56 & 970,95 & 355043,12 \\
\hline Cargabilidade & 31,52 & 8,31 & 0,32 \\
\hline Profundidade & 3,86 & 9,23 & \\
\hline
\end{tabular}

\section{Figura 6: Processamento da SEV 7}

Esta SEV localiza-se na região do gnaisse e apresenta 3 camadas. É importante resaltar que a curva de cargabilidade não foi muito bem ajustada. Poderíamos ter usado um artifício de inverter cada curva separadamente, mas este artifício não foi utilizado, pois consideramos que como a região é a mesma, os dados devem ser processados juntos. Assim não se deu muita relevância aos valores de cargabilidade. Com isso, a primeira camada tem uma resistividade em torno de 400 Ohm.m com cargabilidade em torno de $30 \mathrm{mSeg} . \mathrm{V} / \mathrm{V}$. Razoável identifica-la como um solo de alteração. A segunda camada apresenta resistividade em torno de $1000 \mathrm{Ohm} . \mathrm{m}$ o que poderia ser identificada como um gnaisse mais fraturado ou como o xisto minolítico. Já a terceira camada tem valores de resistividade bem pronunciado e cargabilidade bem baixa, quase zero. Assim identifica-se essa camada como a gnaisse são.

Os dados de Caminhamento Elétrico foram processados no programa RES2DInv. Este é um programa desenvolvido por Geotomo Software Malaya (Geotomo, 2011). O programa gera uma pseudoseção onde cores frias representam corpos condutivos e cores vermelhas corpos resistivos. O dado aqui apresentado pode ser visualizado nas figuras 7 .

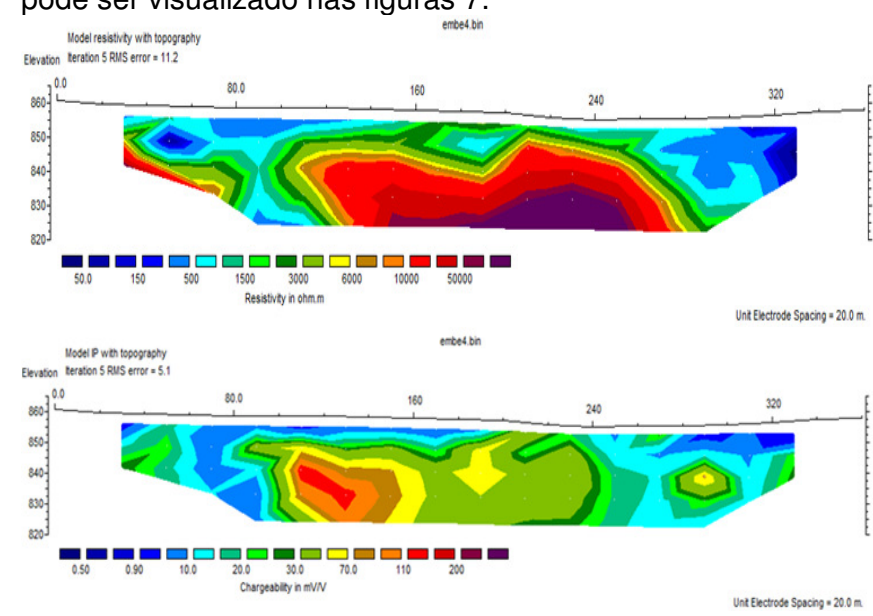

Figura 7: Resultado da inversão para resistividade (primeira figura) e cargabilidade (segunda figura) para o CE4.

No caminhamento 04 notamos que o erro é baixo para ambas pseudoseções. $\mathrm{Na}$ pseudoseção de resistividade notamos que em $85 \mathrm{~m}$ há uma quebra entre uma mesma estrutura resistiva o que indica uma falha geológica nesta localização. A partir de 90m identificamos uma estrutura com resistividade em torno de 50000 Ohm.m o que pode facilmente ser 
correlacionada com o gnaisse. A partir de $240 \mathrm{~m}$ identifica-se uma estrutura mais condutora, iniciando com valores acima de $500 \mathrm{Ohm} . \mathrm{m}$ até valores em torno de 150 Ohm.m o que indicaria o contato do gnaisse com o xisto minolítico rumando para o xisto. Na pseudoseção de cargabilidade notamos uma correlação das estruturas com as conclusões feitas na pseudoseção de resistividade.

Este caminhamento passa pelas SEV's 7 e 6 , nesta ordem. Onde a SEV07 (figura 6) corresponde ao inicio do caminhamento. Na SEV07 foi identificado um gnaisse fraturado ou um xisto minolítico sobre o gnaisse são. E na figura 7 é possível visualizar uma área condutiva com teor intermediário de cargabilidade sobre uma área resistiva sem muita variação nos valores de cargabilidade. Assim corroboramos a hipótese do xisto minolítico sobre o gnaisse possivelmente são. Talvez não encontramos um valor pronunciado marcando o gnaisse devido a própria rocha, pois na SEV07 também não foi possível ajustar a curva de cargabilidade.

\section{Discussão e Conclusões}

Até agora os dados de SEV processados nos permitiram em sua maioria identificar o topo do gnaisse e a espessura do capeamento de solo. Os dados de CE mapearam muito bem o contato entre o gnaisse e o xisto e muitas vezes marcando o xisto minolítico. Favorecendo assim o entendimento do comportamento destas rochas.

Futuramente serão realizados dois perfis de dados de EM34 em dois locais onde foram realizados aquisições de caminhamento elétrico. Se os reseltados entre EM34 e CE forem similares, O EM34 pode ser indicado como um primeiro método a ser aplicado por ser mais rápido e barato. Além disso quando tivermos todos os dados, sera feito um mapa 3D do topo do gnaisse para melhor entender seu compartamento para assim orientarmos as frentes de lavra.

\section{Agradecimentos}

Gostaria de agradecer a CAPES pelo apoio financeiro que vem dando suporte a este trabalho. Também ao meu orientador o prof. Dr. Vargner Roberto Elis que confiou em mim para realizar este trabalho. $E$ que, há anos vem me dando suporte e me ensinando cada dia mais. Por último mas não menos importante, gostaria de agradecer ao IAG/USP por todo o conhecimento e apoio.

\section{Referências}

Bortolozo, C. A., 2011. Inversão Conjunta 1D de dados de SEV e TDEM: Aplicações em hidrogeologia. Dissertação de Mestrado, Universidade de São Paulo.

Elis, V. R., 1998. Avaliação da aplicabilidade de métodos elétricos de prospecção geofísica no estudo de áreas utilizadas para disposição de resíduos. Dissertação de Doutorado. Universidade Estadual Paulista. Rio Claro. Capítulo 3 e 4.
Elis, V. R e Zuquette, L. V., 2000. Aplicação Integrada de Métodos Geoléctricos em Áreas de disposição de Resíduos Industriais: Cidade de Franca, Brasil

Embusa, 2012, Site da empresa EMBU S/A ENGENHARIA E COMÉRCIO: http://www.embusa.com.br, acessado em 30/01/2012

Mineração e Desenvolvimento Sustentável, 2008. Municício de Tenente Ananias/RN. Núcleo de Estudos Sobre o Trabalho Humano da Universidade Federal de Minas Gerais. Secretaria de Geologia, Mineração e Transformação Mineral do Ministério de Minas e Energia. SGM / MME

Moreira, C. A. et al., 2007. Aplicação do método eletromagnético indutivo (EM) no monitoramento de contaminantes em subsuperfície. Revista Brasileira de Geofísica, volume 25 n.4, Outubro/Dezembro 2007. São Paulo.

Santos, A. R. dos, 2002 Geologia de Engenharia: Conceitos, Método e Prática. São Paulo. Capítulo I e casos 2, 6, 12 e 18 .

Telford, W. M., Gerald, L. P., Sheriff, R. E., 1990. Applied Geophysics, Cambridge University Press, $2^{\circ}$ edição. Cambridge University Press 860p.

Valverde, Fernando M., et al, 2001. Balanço Mineral Brasileiro. Capítulo: Agregado para a Construção Civil páginas 1-15. Disponível no site http://www.dnpm.gov.br/portal/assets/galeriaDocumento/ BalancoMineral2001/agregados.pdf acessado no dia 02/02/2012. 


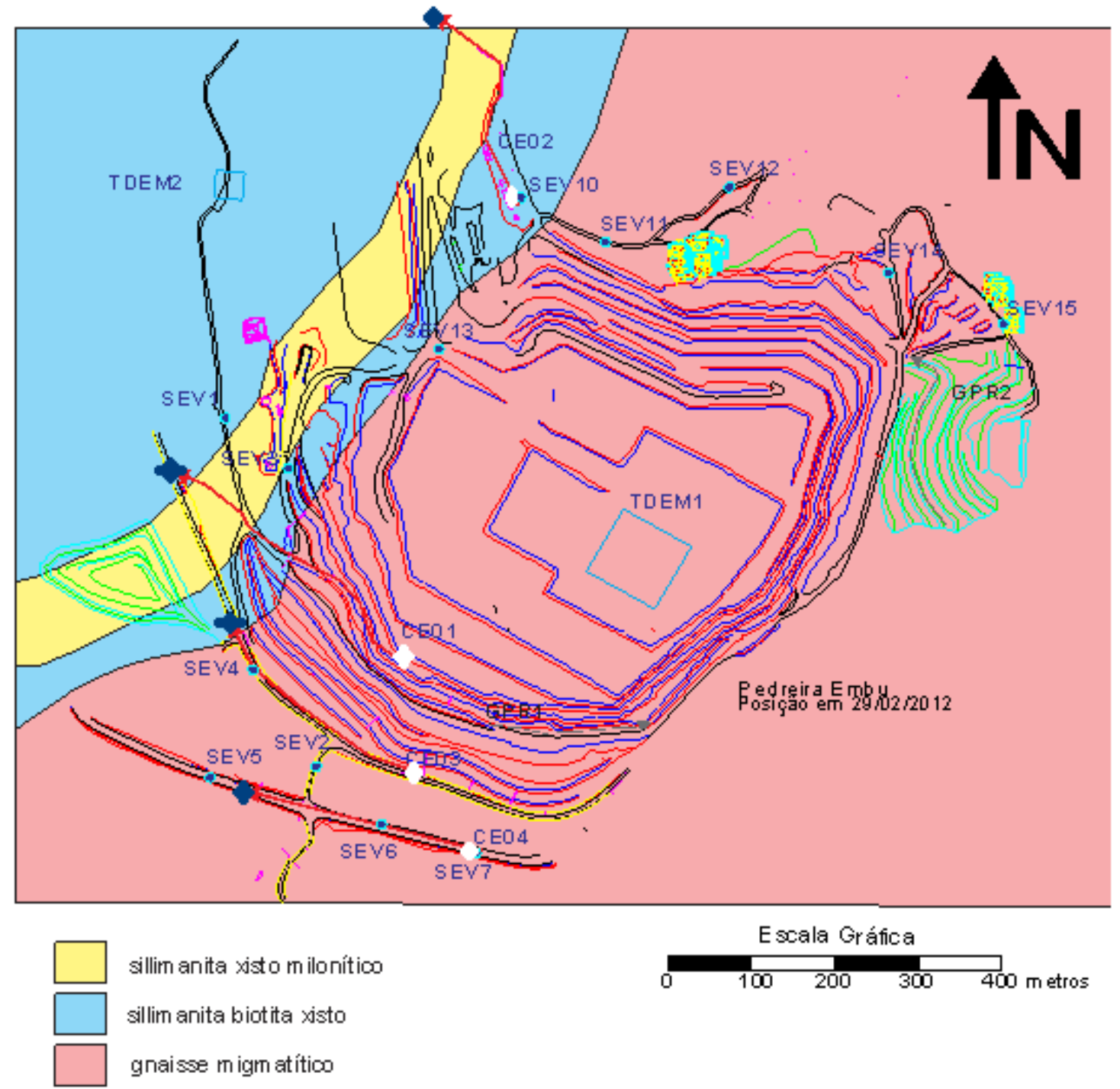

Figura 3: Disposição dos dados coletados, mostrando as 13 SEV's adquiridas e os 4 CE. As marcações em branco indicam o início do CE e em azul o final. 